\title{
REGIONS OF EXCLUSION FOR THE LATENT ROOTS OF A MATRIX
}

\section{HANS SCHNEIDER}

The well-known Theorem 0 is due to S. Gersgorin [2] and A. Brauer [1]:

Theorem 0. Let $A:\left[a_{i j}\right]$ be an $n \times n$ matrix with complex elements. Then the latent roots of $A$ lie in the union of the $n$ circular regions $\left|z-a_{i i}\right| \leqq P_{i}, i=1, \cdots, n$, in the complex plane, where $P_{i}=\sum_{j}^{\prime}\left|a_{i j}\right|$ and $\sum_{j}^{\prime}$ denotes summation from $j=1$ to $j=n$ with $j=i$ omitted.

It is the purpose of this note to point out that there may also exist bounded regions which exclude the latent roots of $A$.

Let $B:\left[b_{i j}\right]$ be an $n \times n$ matrix with complex elements. Let $(\mu(1), \cdots, \mu(n))$ be a permutation of $(1, \cdots, n)$, and let $B^{\prime}:\left[b_{i j}^{\prime}\right]$ be the matrix $\left[b_{i j}^{\prime}\right]=\left[b_{i \mu(j)}\right]$. Thus $B^{\prime}$ is obtained from $B$ by a permutation of its columns, and therefore $B$ is nonsingular when $B^{\prime}$ is nonsingular.

The matrix $B^{\prime}$ is nonsingular if

$$
\left|b_{i i}^{\prime}\right|>\sum_{j}^{\prime}\left|b_{i j}^{\prime}\right|, \quad \text { for } i=1, \cdots, n,
$$

(cf. e.g. O. Taussky [5]), and so $B$ is nonsingular when

$$
\left|b_{i \mu(i)}\right|>\sum_{j}^{\prime}\left|b_{i \mu(j)}\right|=\sum_{j \neq \mu(i)}\left|b_{i j}\right|, \quad \text { for } i=1, \cdots, n .
$$

Now let $B=\lambda I-A$, where $\lambda$ is a latent root of $A$. Then $b_{i i}=\lambda-a_{i i}$, and $b_{i j}=-a_{i j}$, when $i \neq j$. Since $B$ is singular not all the inequalities (1) can hold. Hence there is an $i, 1 \leqq i \leqq n$, such that either

$$
\left|\lambda-a_{i i}\right| \leqq P_{i} \text { and } i=\mu(i),
$$

or

$$
\left|a_{i \mu(i)}\right| \leqq\left|\lambda-a_{i i}\right|+\sum_{j}^{\prime \prime}\left|a_{i j}\right| \text { and } i \neq \mu(i),
$$

where $\sum_{j}^{\prime \prime}$ denotes summation from $j=1$ to $j=n$, with $j=i$ and $j=\mu(i)$ omitted. We now immediately obtain Theorem 1 .

Theorem 1. Let $A$ be an $n \times n$ matrix with complex elements. Let $(\mu(1), \cdots, \mu(n))$ be a permutation of $(1, \cdots, n)$. Then the latent roots of $A$ lie in the union of the $n$ regions

Received by the editors July 21, 1953. 


$$
\begin{array}{ll}
\left|z-a_{i i}\right| \leqq P_{i} \text { when } \quad i=\mu(i), \\
\left|z-a_{i i}\right| \geqq Q_{i} \text { when } \quad i \neq \mu(i),
\end{array}
$$

where $Q_{i}=\left|a_{i \mu(i)}\right|-\sum_{j}^{\prime \prime}\left|a_{i j}\right|$.

If $\mu(i)=i$ when $i=1, \cdots, n$ then Theorem 1 reduces to Theorem 0 . In this case we obtain a bounded region in the complex plane within which all latent roots of $A$ lie. If some $i \neq \mu(i)$, Theorem 1 may yield a bounded region of the complex plane within which no latent root of $A$ can lie. Let $I$ be the union of the interiors and boundaries of the circles $\left|z-a_{i i}\right|=P_{i}$ when $i=\mu(i)$. Let $C I$ be the complement of $I$. Let $E$ be the intersection of the interiors of the circles $\left|z-a_{i i}\right|=Q_{i}$ when $i \neq \mu(i)$, such an interior being empty when $Q_{i} \leqq 0$. It is easily seen that the region of exclusion for the latent roots of $A$ given by Theorem 1 is the intersection of $C I$ and $E$. If $E$ is empty, or if $E$ is contained in $I$, there will be no region of exclusion. In particular, there is no region of exclusion if $Q_{i} \leqq 0$ for some $i \neq \mu(i)$.

We shall apply Theorem 1 to the matrix

$$
A=\left[\begin{array}{rrrr}
1 & 4 & 1 & 0 \\
4 & 2 & 0 & 0 \\
0 & 1 & -1 & 2 \\
1 & 0 & 3 & -2
\end{array}\right] .
$$

(In the case of nondiagonal elements only the absolute values are relevant.) From the permutation $(1,2,3,4)$ it follows by means of Theorem 1 (or Theorem 0 ) that the latent roots of $A$ lie within or on the circles $|z-1|=5$ or $|z+2|=4$. From the permutation $(2,1,3,4)$ it follows that no latent root of $A$ lies in that part of the interior of the circle $|z-1|=3$ which is outside $|z+2|=4$. From the permutation $(2,1,4,3)$ it follows that there is no latent root of $A$ within $|z+1|=1$. No region of exclusion is obtained from $(1,2,4,3)$ even though $Q_{3}$ and $Q_{4}$ are positive.

If $n=2$ the only possible permutations of $(1,2)$ are $(1,2)$ and $(2,1)$. Let $\lambda$ be a latent root of the $2 \times 2$ matrix $A$. Applying Theorem 1 with the permutation $(1,2)$ we obtain that $\lambda$ lies within or on one of the circles

$$
\left|z-a_{11}\right|=\left|a_{12}\right|, \quad\left|z-a_{22}\right|=\left|a_{21}\right| .
$$

When Theorem 1 is used with the permutation $(2,1)$ it follows that $\lambda$ lies outside or on one of the circles (2). Thus $\lambda$ lies inside or on one of the circles (2), but not inside both. This is a slightly weakened form of a theorem due to O. Taussky [6]. 
Some other known results may be generalized in the same way as Theorem 0. P. Stein has proved that if $B^{\prime}$ is singular and its latent root 0 has $m$ associated linearly independent latent column vectors, then at least $m$ of the inequalities

$$
\left|b_{i i}^{\prime}\right| \leqq \sum_{j}^{\prime}\left|b_{i j}^{\prime}\right|, \quad i=1, \cdots, n,
$$

are satisfied (P. Stein [4], O. Taussky [7]).

The latent column vectors of $B^{\prime}$ associated with 0 are just those of $B$ after the permutation $(\mu(1), \cdots, \mu(n))$ has been applied to the indices of the elements of the latter. Hence $B$ has precisely as many linearly independent latent column vectors associated with 0 as $B^{\prime}$. If we now apply the argument leading to Theorem 1 to Stein's result we obtain Theorem 2 .

TheOREM 2. Let $\lambda$ be a latent root of $A$ which has $m$ linearly independent latent column vectors associated with it. Let $(\mu(1), \cdots, \mu(n))$ be a permutation of $(1, \cdots, n)$. Then $\lambda$ lies in at least $m$ of the regions

$$
\begin{aligned}
& \left|z-a_{i i}\right| \leqq P_{i}, \text { when } i=\mu(i), \\
& \left|z-a_{i i}\right| \geqq Q_{i}, \text { when } i \neq \mu(i) .
\end{aligned}
$$

Results which apply to irreducible matrices only do not seem to be extendable in this simple manner. The reason is that $B^{\prime}$ may be reducible when $A$ and $B=\lambda I-A$ are irreducible. It is possible to apply the above method to more general bounds for the latent roots of a matrix, such as those of Ostrowski [3], but the regions of exclusion obtained can apparently not be expressed in a simple form.

\section{REFERENCES}

1. A. Brauer, Limits for the characteristic roots of a matrix, I, Duke Math. J. vol. 13 (1946) pp. 387-395.

2. S. Gersgorin, Ueber die Abgrenzung der Eigenwerte einer Matrix, Izvestia Akademii Nauk SSSR vol. 7 (1931) pp. 749-754.

3. A. Ostrowski, Ueber das Nichtverschwinden einer Klasse von Deierminanten und die Lokalisierung der charakteristischen Wurzeln von Matrizen, Compositio Math. vol. 9 (1951) pp. 209-226.

4. P. Stein, $A$ note on the bounds of multiple characteristic roots of a matrix, Journal of Research, National Bureau of Standards vol. 48 (1952) pp. 59-60.

5. O. Taussky, A recurring theorem on determinants, Amer. Math. Monthly vol. 56 (1949) pp. 672-676.

6. - Bounds for the characteristic roots of matrices, Duke Math. J. vol. 15 (1948) pp. 1043-1044.

7. - Bounds for the characteristic roots of matrices II, Journal of Research, National Bureau of Standards vol. 46 (1951) pp. 124-125.

The Queen's University, Belfast 